\title{
(C) OPEN ACCESS \\ Palliative care research centre's \\ move into social media: constructing a framework for ethical research, a consensus paper
}

\author{
Noreen Hopewell-Kelly, ${ }^{1}$ Jessica Baillie, ${ }^{2}$ Stephanie Sivell, ${ }^{1}$ Emily Harrop, ${ }^{1}$ \\ Anna Bowyer, ${ }^{3}$ Sophia Taylor, ${ }^{1}$ Kristen Thomas, ${ }^{1}$ Alisha Newman, ${ }^{1}$ \\ Hayley Prout, ${ }^{1}$ Anthony Byrne, ${ }^{1}$ Mark Taubert, ${ }^{4}$ Annmarie Nelson ${ }^{1}$
}

\begin{abstract}
${ }^{1}$ Marie Curie Palliative Care Research Centre, Institute of Cancer and Genetics, Cardiff University School of Medicine, Cardiff, UK

${ }^{2}$ School of Healthcare Sciences, College of Biomedical and Life Sciences, Cardiff, UK

${ }^{3}$ Community Palliative Care Services Team, Ty Hafan, Vale of Glamorgan, UK

${ }^{4}$ Palliative Care Department, Velindre Cancer Centre, Cardiff, UK
\end{abstract}

\section{Correspondence to}

Dr Annmarie Nelson, Marie Curie Palliative Care Research Centre, Institute of Cancer and Genetics, Cardiff University, School of Medicine, Neuadd Meirionnydd, Heath Park, Cardiff, CF14 4YS, UK; nelsona9@cardiff.ac.uk

Received 12 March 2015 Revised 12 October 2015 Accepted 16 December 2015 Published Online First 28 January 2016

\section{A Check for updates}

To cite: Hopewell-Kelly N, Baillie J, Sivell S, et al. BMJ Supportive \& Palliative Care 2019:9:219-224.

\section{ABSTRACT}

Background Social media (SM) have altered the way we live and, for many, the way we die. The information available on even the rarest conditions is vast. Free from restrictions of mobility, time and distance, SM provides a space for people to share experiences of illness, death and dying, and potentially benefit from the emotional and practical support of others $n$ similar positions. The communications that take place in these spaces also create large amounts of 'data' which, for any research centre, cannot be ignored. However, for a palliative care research centre the use of this 'data' comes with specific ethical dilemmas.

Methods This paper details the process that we, as a research, went through in constructing a set of ethical guidelines by which to work. This involved conducting two consensus days; one with researchers from within the centre, and one with the inclusion of external researchers with a specific interest in SM.

Results The primary themes that emerged from the consensus meetings includes; SM as a public or private space; the status of open and closed groups; the use of historical data; recruiting participants and obtaining informed consent and problems of anonymity associated with dissemination.

Conclusions These are the themes that this paper will focus on prior to setting out the guidelines that we subsequently constructed.

\section{INTRODUCTION}

The online world is alive with the topics of illness, dying and death ${ }^{1} 2$ and for a palliative care research centre this wealth of communication has implications for research practices. In a recent paper 'Using social media in supportive and palliative care research', ${ }^{3}$ its authors present a detailed overview of how social media (SM) have evolved as a tool for research within the field of palliative care, but stops short of making any recommendations with regard to ethical and moral stances. It concludes with a call for ethical guidelines to be constructed for the use of SM. This paper details the processes that we followed when initiating the construction of our own set of ethical guidelines.

\section{BACKGROUND}

SM can be defined as any 'web-based applications that allow people to create and exchange content'. ${ }^{3}$ In this sense, SM can be understood to include blogs, microblogs, internet forums and social networking sites. ${ }^{3}$ The benefits of SM as a research tool are potentially vast and very tempting; they enable the opportunity to cross spatial and time barriers that exist in traditional research, they afford direct access to potential research participants, and provide a means of accessing vast amounts of data, quickly and efficiently. As an academic unit attached to a clinical trials unit, our research centre is committed to mixed methods research, and it has become clear that SM are an area of research rich with potential, but one which is also laden with ethical and practical challenges. Whereas guidance on the use of SM has been issued for healthcare professionals, ${ }^{4-6}$ which takes a view of SM as 'new circumstances in which established principles apply', 5 no clear guidance currently exists for researchers interested in using SM.

Recent papers exploring the use of SM in supportive and palliative care have 
identified both the extent of interest in palliative and end-of-life care on the widely used 'twitter' site, ${ }^{2}$ as well as key issues involved in SM research on this topic. $^{3}$ It is argued that SM is not an unproblematic research opportunity but presents great challenges including: recruitment difficulties, data ownership, sample bias and screening problems. ${ }^{3}$ These authors took steps to explore these complex challenges, primarily in relation to the recruitment of research participants in an SM environment. In their conclusion, they called for ethics committees and SM researchers to recognise their responsibility to work in SM as they would in the 'real world', within strong ethical guidelines that ensure good working practice in all research. A literature search around the use of SM in research was conducted and several articles were identified that contained generic proposals for ethical guidelines in SM research. ${ }^{7-9}$ No definitive principles were identified, therefore, this discussion paper has taken tentative steps to construct a set of research guidelines by which to conduct ethical palliative research in the SM environment.

\section{Consensus days}

Our research centre conducted two consensus sessions, which were constructed to discuss and debate the complex ethical challenges embedded in SM research. The first session involved seven researchers from within the unit who had a range of experiences of using SM sites for personal and professional purposes; usually, dissemination of team activities, although with no prior experience of using such sites to carry out research. The discussion was guided by questions generated by a review of the literature, which was conducted by the group facilitator and circulated to participants in advance of the first meeting. The second meeting further explored the issues raised in the first session, and also included external researchers with a strong background in SM research. Our concern did not mirror the recruitment focus of Comabella and Wanat, ${ }^{3}$ but instead reflected those issues raised in Zimmer's work ${ }^{9}$ around the ethics of using SM, specifically in relation to privacy and anonymity. This meant that the two consensus-day discussions placed far greater emphasis on establishing our position over the debated status of the internet and internet data (public/private), which encompassed a focus on the intentions of SM users, and how these should be incorporated into what we considered to be ethical research. Considerable debate and discussion took place during these consensus meetings, addressing the pros and cons of SM research. An overview of guidelines constructed for the conduct of ethical internet research informs the conclusion of the paper.

\section{DEBATE AND CONSENSUS: THE KEY ISSUES}

This section describes the key issues identified and debated in the literature and the consensus group discussions, organised under the headings: the internet, a public or private space?; the status of open and closed groups; use of historical data; recruiting participants and obtaining informed consent; anonymity in dissemination.

\section{The internet, a public or private space?}

The spatial way in which the internet is viewed has far-reaching consequences on the way that the intentions of the SM user are perceived and any research subsequently conducted. The questionable spatial status of the internet is widely debated and no distinction can easily be drawn between what can be considered public and that which is perceived as private. The accessibility of online communities has been likened to that of television or newspaper interviews ${ }^{10}$ which suggests that online communities can be considered to reside in the public domain. The journalistic media certainly do not hesitate in publishing publicly available microblog uploads from celebrities and politicians, and comments from the general public on healthcare debates are used in numerous articles and reports. Alternatively, the expectations of where and what internet users are communicating is considered to be the dominant ethical consideration for researchers. ${ }^{10}$ Comabella and Wanat $^{3}$ addressed this point when they highlighted a subtle difference between the internet forum and other SM platforms such as YouTube or Twitter where the latter are created with the intention of reaching a large public audience. However, this can be contrasted with Frankel and Siang's position, that although communications may be easily accessible, many SM users would not consider their posts as potential research material, and accordingly, for research purposes, their posts should be considered private. ${ }^{10}$

Further complicating this debate are those scholars who suggest SM be approached as neither public nor private, but instead, a space that exists somewhere between the two; a 'publicly private' and 'privately public' space. Moreover, it can be argued that the internet should not be viewed exclusively as a site of human communication and interpersonal interaction but as a means of cultural production that can be considered in its textual form. ${ }^{11}$ In line with that approach, researchers need not consider the internet in 'spatial' terms at all but can, instead, focus on the dialogue that is extracted from the internet as text that is available for reproduction and public consumption. Concerns with the text-based approach can, nevertheless, be seen in the need for researchers to navigate their way through issues of ownership, removal and copyright, ${ }^{11}$ however, the considerations seen in spatial arguments are less apparent within this framework.

The way in which SM are understood, be they public/private and/or text based, will have a direct impact on the way that researchers frame the position 
of the participants themselves, and this will, accordingly, shape the way that the research is conducted in its entirety. ${ }^{3}$ The impact of these spatial/textual discourses is, therefore, embedded in subsequent methods of recruiting participants; approaches to obtaining informed consent; ensuring confidentiality; the questionable need for authenticating participants' identities and the data they construct; and dissemination of the work that retains participants' confidentiality. Comabella and Wanat ${ }^{3}$ urge researchers to establish their position in relation to these points prior to beginning research in order to ensure the protection and privacy of participants.

Our consensus groups also considered the spatial position of the internet to be crucial to the way that the researcher would perceive the forum they were approaching, the users of that forum and the data that could potentially be drawn from that particular form of SM. This meant that the questionable spatial status of closed groups, open groups, historical posts and bloggers were issues that all required individual discussion, as were the intentions of the SM within those groups.

\section{Status of open and closed groups}

Closed groups were defined as those that a user needed to join prior to gaining access (such as Facebook, user support groups and so on). This kind of group was initially considered to occupy a private space for two reasons:

- Owing to the privacy settings that administrators/users can employ to make communications available to a specific group of people;

- Because users within closed groups are liable to consider their communications private and will conduct their posts accordingly.

However, contrary to the idea that privacy settings create a private forum for its users, changes to security settings implemented by internet providers (eg, Facebook, Yahoo and so on) necessitate that both users and group administrators maintain constant awareness of the security status of the groups to which they belong. It would be naive to presume that this is undertaken by all SM users, and thus, closed and private groups may at different junctures become more accessible to a public audience. Similarly, the necessity to join a group does little in the way of creating a barrier to public access as anyone, regardless of their appropriateness to the group, can create an identity with which to join the online forum and view the 'private' communications with relative ease. The true extent to which any 'private' communications can be considered to take place in a private space is, therefore, once again raised. Closed groups would, for this reason, be best understood to occupy a perceived private space that is potentially vulnerable to a public audience.

Open access forums were considered by the consensus groups to be firmly grounded in the public space.
Bloggers were perceived to be focused on disseminating their communications to a public audience, and for that reason their posts are widely accessible to a public and sometimes publicised space. It is important to recognise that a perceived intent for public dissemination does not automatically equate to a willingness to become a research participant, thus whether it is ethical to reposition this data in such a way remains arguable. An alternative approach to the public/ private debate is the textual approach described above which suggests that spatial concerns about the internet could be avoided if one considered SM communications as neither public nor private, but instead as textual data. The consensus groups largely discussed this approach in relation to the use of historical data on the internet.

\section{Use of historical data}

Across the consensus delegates it was agreed that the text-based approach enables internet communications to be repositioned as text that stands alone from its author. Theoretically, it can be argued that text is not vulnerable to the same person-centred concerns that spatial perceptions involve, and thus, the text-based approach avoids complicated ethical quandaries. In support of this approach, one can identify parallels with its physical world equivalent where researchers draw from previously conducted research; however, physical world research is not vulnerable to the same complexities as that conducted online where cyber data is traceable to its original forum and author. Although a textual approach theoretically circumnavigates person-centred concerns (as it is simply text that is being discussed), for us there remains a discomfort about removing the authors from the text, and thus, concerns about anonymity and consent remained unresolved.

Illustrating this point was a concern around the lack of consent in the textual approach to historical data (or any other forum). It was suggested that in the equivalent 'real world' historical research, there would have been a consent process undertaken in the collection of the original material. That consent could, in principle, be seen to extend to the use of the data in future research projects. However, for a text-based approach in SM research where there is no original consent process, a physical world equivalent cannot be drawn; subsequently, the (internet) author's intentions and wishes would remain unknown. It would seem fair to presume that people do not communicate across SM with any consideration that their communications might be repositioned as data, and used for research purposes. Subsequently, out of consideration for the author and the sensitivity of the communications of interest in palliative research, the consensus groups concluded that a textual approach does not avoid the person-centred complexities embedded in the spatial perspective, and therefore, 
should not be considered an appropriate approach or way forward.

\section{Recruiting participants and obtaining informed consent}

Online recruitment can be achieved in two primary ways; prospectively, with the researcher making contact with participants directly, or retrospectively, with the researcher making a general request to an internet forum/wall for people to opt out if they do not wish to be included in the study. Both approaches are problematic; locating valid contact details for the recruitment of prospective participants can be an excessively time-demanding process, and due to the fluidity of the internet forum (where community users come and go), people may not be aware of retrospective recruitment relating to their online communications. ${ }^{7}$ In palliative research, retrospective consent is particularly problematic due to the likelihood that the person may no longer be alive. The method used to recruit participants will have a subsequent impact on the way that informed consent is viewed and approached.

Comabella and Wanat ${ }^{3}$ suggest that the direct recruitment of participants may not always be appropriate or necessary, and they cite the use of data from blogs and YouTube as evidence of this. We took this a step further and considered whether this would be the case where the researcher spends a period of time passively observing online communities (lurking). As previously suggested, where the internet is understood as a public domain, then postings and communications may be considered 'fair research game" ${ }^{12}$ that do not require specific consent from community members. However, legal issues relating to ownership of data stored on the servers of private companies complicate this matter, ${ }^{3}$ and the sensitive nature of palliative research might make it ethically appropriate to seek consent from all research participants, regardless of the status of the space in which their communications took place. ${ }^{7} 11{ }^{12}$ Where/if this is deemed to be the case, a comprehensive systematic process must be constructed in order to gain informed consent from online participants that aims to overcome the challenges embedded in this process.

The process of consent in the physical world involves researchers ensuring that participants receive all relevant information, (normally in the form of paper/electronic copies of information sheets) and making efforts to check that they have a satisfactory understanding of that information. Attempts to ensure that participation is voluntary, and to clarify comprehension of the study, can be measured to some degree by physical cues and markers; however, there is no equivalent of this in gaining online consent. The equivalent of a signature on a consent form would be the click of a box next to a statement agreeing to consent to the study, ${ }^{10}$ yet people regularly click statements of agreement without having read the information provided, and thus, the true extent to which their consent is 'informed' is difficult to establish.

The delegates in our groups were in agreement that researchers have a duty of care in the online research process, and it is our responsibility to ensure that this is in place, is appropriately adhered to (ensuring possible harms are avoided), and that our data-gathering intentions are transparent. For this reason, the delegates of our consensus meetings were in agreement that although SM could and indeed should be used as a research tool, its spatial position would best be understood to straddle both public and private spheres; private in that communications may have been constructed with a perception of privacy; public in that those communications are liable to be publically accessible. For this research centre, this means that the privacy of internet users should be a significant consideration in the conduct of SM research, and should therefore involve consenting internet users prior to the collection of their posts for research purposes. Where this is not possible, the research centre felt that the appropriateness of its use should be seriously questioned.

Creating online forums

With this in mind, we considered whether it would be beneficial to construct our own online forum in which to conduct research. Comabella and Wanat ${ }^{3}$ suggested 10 steps to maximise participant recruitment in SM including the creation of Facebook/Twitter pages for research recruitment purposes. We would suggest that there is a very real potential to not only recruit across $\mathrm{SM}$ in this way, but to construct and conduct the research in its entirety within that SM forum. The construction of this research forum would not only benefit from a more simplistic consent procedure, but transparency in the research process would also be far easier to achieve. Users would be clear about the reason for the group's existence, and they would be aware that all their posts/communications were to be viewed as potential research data; importantly, they would know this prior to the communications taking place.

Although this would be an artificial and staged forum which, subsequently, may be unable to replicate the natural flow of communications constructed on other forms of SM, this scenario could be likened to the staged environment of the focus group in the physical world, an environment that is considered to be an enduring and beneficial research method. Counter arguing this drawback are also the benefits that can be drawn from having a discussion group that is not being led by the researcher (in the way that they would lead an interview); thus, regardless of the staged research environment, we believe that an element of naturalness could be achieved in the discussion forum. 


\section{Anonymity in dissemination}

When composing reports, qualitative research tends to draw heavily from the original transcripts (the SM equivalent being text from blogs/forum pages and so on), which is usually presented in the form of quotes. This is unproblematic in physical world research where the potential for identifying participants should be minimal; but for internet data, where there exists a very real potential for quotes to be traced back to the original author, it is considered to be an issue of significant concern. Three potential ways of approaching this problem were discussed in our consensus meetings:

- Rewrite what people say;

- Ensure the potential for identification is disclosed in the consent process;

- Consider the construction of online identities to be sufficient barrier to identification.

The benefit of rewriting online communications is that the original communications and forum cannot be traced through a search engine, thus, a degree of distance can be afforded between the text and the author. Although some researchers may not perceive this to be a problematic way of dealing with data, it can be argued that removing data from its original frame and rewriting it to suit research requirements will obliterate the original impact of the experience that was narrated, albeit in a textual form. ${ }^{13}$ This mirrors Ricoeurs concept of distanciation where every retelling, rewriting and reframing of the story creates an increasing distance from the essence, meaning and impact of the person's actual experience. ${ }^{13}$ As a research centre, we are keen to remain sensitive to the nature of our palliative research and want to ensure that, as far as possible, participants' experiences are not lost within the reframing and retelling of their communications. For these reasons, the consensus meeting drew the conclusion that where possible, distanciation should be kept to a minimum and, instead, participants should be made aware of the potential for identification within the consent process.

Rather than attempting to avoid the issue of anonymity, it was suggested that it may be more appropriate to highlight the problem within the consent process, giving the participant the opportunity to decide the extent to which their online identity needs protecting, and thus, giving them the time to respond appropriately to their decision. It was also suggested that where there was a forum created specifically for research purposes, participants could create an identity that was unique to that forum alone. However, the creation of multiple identities is not always permitted across SM forums, and as such, this may not be a permissible means with which to proceed. Subsequently, our researchers conceded that issues of anonymity needed to be highlighted in the consent process, but online identities would then be considered to provide a sufficient level of anonymity to the participant's real-world identity.

\section{GUIDELINES}

The discussions that have been detailed within this paper are reflected in the guidelines that we have constructed to inform qualitative and mixed method studies in palliative care research, using online data. The complex and lengthy discourse embedded in constructing ethical guidelines for SM research remain in their infancy; we, accordingly, invite further discussion and debate in this ongoing challenge. Nevertheless, to summarise the guidelines as they currently stand:

- Internet discussions should be considered as private, regardless of the forum in which they have been constructed;

- A text-based approach to SM research is not considered an appropriate method in the palliative research of this research centre;

- Recruitment of participants should be transparent with adequate opportunity for participants to ask questions;

- Consent should always be taken from participants for the use of their communications/postings in ways that deviate from the posters' assumed original intentionsfor example, research;

- Information sheets that are clear about confidentiality issues should be provided;

- Anonymity issues in dissemination of research must be highlighted to participants;

- Online identities are considered to be a sufficient safeguard to participant anonymity, however, the importance of online identities is recognised by the research centre;

- The use of historical text is considered to be problematic, and therefore, not encouraged;

- Closed forums created specifically for research purposes are considered to be the most appropriate way to conduct palliative research online.

\section{LIMITATIONS AND IMPLICATIONS FOR FUTURE RESEARCH STUDIES}

Our discussions were primarily focused on the use of personal opinion or disclosure by internet posters, usually in the form of free text that has the potential to be 'mined' as qualitative data, and the specific challenges that this brings in terms of ensuring anonymity and protecting participants' identities. We recognise that other more quantitative approaches to online data may not carry these risks and might reasonably and appropriately make use of SM data without obtaining individual consent, for example. These guidelines are most applicable to qualitative approaches, and researchers using other methodologies will need to use their judgement when interpreting them.

There may also be biases caused by the composition of our groups. Our groups included a mix of researchers and researcher-clinicians with varying degrees of 
personal and professional involvement, familiarity and interest in SM sites. While this enabled a range of experiences, and perspectives to be considered, patient and carer perspectives on potential uses of their online communications were absent from these discussions. A proposed next step in our work in this area is to seek consensus on our guidelines from a much wider group of participants, including patient and carer users of SM sites, using an online consensus-building methodology such as a Delphi Survey. This would also enable us to pilot our proposed idea for 'closed forums' in online research.

\section{CONCLUSION}

Using SM in palliative research creates complex challenges that require extensive consideration. This paper has detailed the initial discussions taken by our research centre around using SM in its research. These discussions have culminated in a set of guidelines that we are now looking to pilot and further develop. In practice, these will take some steps towards ensuring that SM research within our palliative research centre is conducted in an agreed and ethically acceptable way.

Twitter Follow Annmarie Nelson at@annmarienelson0

Contributors NH-K and AN were responsible for conception of the study and its subsequent design. NH-K and JB were responsible for facilitating the consensus meetings, with NH-K primarily responsible for analysing and reporting the outcomes of the meetings. JB, SS, ST, KT, AB, AN and HP attended the consensus meetings. NH-K was responsible for manuscript writing; JB, SS, AB, ST, KT, AN, HP, MT and AN provided comments on the drafts of this paper and approved the final version.

Funding The work is supported by core funding from the Marie Curie Palliative Care Research Centre based at Cardiff University School of Medicine (grant reference:

MCCC-FCO-14-C). The study was conceptualised and overseen by core members of the Marie Curie team at Cardiff.

Competing interests None declared.

Provenance and peer review Not commissioned; externally peer reviewed.
Data sharing statement All data from the consensus days are available on request.

Open Access This is an Open Access article distributed in accordance with the Creative Commons Attribution Non Commercial (CC BY-NC 4.0) license, which permits others to distribute, remix, adapt, build upon this work noncommercially, and license their derivative works on different terms, provided the original work is properly cited and the use is non-commercial. See: http://creativecommons.org/licenses/by$\mathrm{nc} / 4.0 /$

\section{REFERENCES}

1 Taubert M, Watts G, Boland J, et al. Palliative social media. BMJ Support Palliat Care 2014;4:13-8.

2 Nwosu AC, Debattista M, Rooney C, et al. Social media and palliative medicine: a retrospective 2-year analysis of global twitter data to evaluate the use of technology to communicate about issues at the end of life. BMJ Support Palliat Care 2015;5:207-12.

3 Comabella CCI, Wanat M. Using social media in supportive and palliative care research. BMJ Support Palliat Care 2015;5:138-45.

4 British Medical Association. Using social media: practical and ethical guidance for doctors and medical students. London: British Medical Association, 2011.

5 General Medical Council. Doctors use of social media. London: General Medical Council, 2013.

6 Nursing and Midwifery Council. Guidance on using social media responsibly. London: Nursing and Midwifery Council, 2015.

7 Eysenbach G, Till JE. Ethical issues in qualitative research on internet communities. BMJ 2001;323:1103-5.

8 Moreno MA, Fost NC, Christakis DA. Research ethics in the MySpace era. Pediatrics 2008;121:157-61.

9 Zimmer M. "But the data is already public" On the ethics of research in Facebook. Ethics Inf Technol 2010;12:313-25.

10 Frankel MS, Siang S. Ethical and legal aspects of human subjects research on the Internet. AAAS online 1999.

11 Bassett E, O’Riordan K. Ethics of Internet research: contesting the human subjects research model. Ethics Inf Technol $2002 ; 4: 233-47$.

12 Flicker S, Haans D, Skinner H. Ethical dilemmas in research on Internet communities. Qual Health Res 2004;14:124-34.

13 Czarniawska B. Narratives in social science research. Sage, 2004. 